\title{
Disturbances in a VLF radio signal prior the $M=4.7$ offshore Anzio (central Italy) earthquake on 22 August 2005
}

\author{
P. F. Biagi ${ }^{1,2}$, L. Castellana ${ }^{1}$, T. Maggipinto ${ }^{1}$, D. Loiacono ${ }^{1}$, V. Augelli $^{1}$, L. Schiavulli ${ }^{1}$, A. Ermini ${ }^{3}$, V. Capozzi $^{4}$, \\ M. S. Solovieva ${ }^{5}$, A. A. Rozhnoi ${ }^{5}$, O. A. Molchanov ${ }^{5}$, and M. Hayakawa ${ }^{6}$ \\ ${ }^{1}$ Department of Physics, University of Bari, Via Amendola, 173 - 70126 Bari, Italy \\ ${ }^{2}$ Inter-Department Centre for the Evaluation and Mitigation of the Volcanic and Seismic Risk, University of Bari, Italy \\ ${ }^{3}$ Department of Engineering of Enterprise, University of Roma Tor Vergata, Via di Tor Vergata - 00133 Rome, Italy \\ ${ }^{4}$ Department of Biomedical Sciences, University of Foggia, Via L. Pinto-71100 Foggia, Italy \\ ${ }^{5}$ United Institute of the Earth's Physics, Russian Academy of Science, Bolshaya Gruzinskaya 10, 123995 Moscow, Russia \\ ${ }^{6}$ Department of Electronic Engineering, The University of Electro-Communications, 1-5-1 Chofugaoka, Chofu City, Tokyo \\ 182-8585, Japan
}

Received: 20 June 2008 - Revised: 28 August 2008 - Accepted: 28 August 2008 - Published: 10 October 2008

\begin{abstract}
On 22 August 2005 an earthquake with magnitude $M=4.7$ occurred in the Anzio (central Italy) offshore area. From 2002, a VLF-LF radio receiver is into operation in Bari (southern Italy). The intensity and the phase of the signals transmitted by $\mathrm{GB}(f=16 \mathrm{kHz}$, United Kingdom $)$, FR $(f=20.9 \mathrm{kHz}$, France), GE ( $f=23.4 \mathrm{kHz}$, Germany), IC $(f=37.5 \mathrm{kHz}$, Island) and IT ( $f=54 \mathrm{kHz}$, Sicily, Italy) has been monitored with a $5 \mathrm{~s}$ sampling rate. The previous epicenter is near enough to some of the radio paths and the data collected were studied in order to reveal possible seismic effects. The raw analysis revealed a clear drop in the intensity of the FR radio signal on 19 August. Then the wavelet analysis was applied to the intensity and the phase data of the different radio signals. In the mentioned day an increase in the band 60-120 min was revealed in the spectra of the FR signal. Then the principal component analysis was applied and again the 19 August stood up as an anomalous day for the FR radio signal. The path of this signal, among the paths of the five radio signals collected by the Bari receiver, is the nearest to the mentioned epicentre and the anomaly revealed on 19 August appears as a precursor of the earthquake. This result confirms the possible precursor revealed by other researchers in the air Rn content in a site located 5 miles far from the epicenter.
\end{abstract}

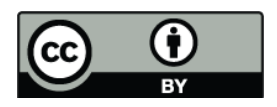

Correspondence to: P. F. Biagi

(biagi@fisica.uniba.it)

\section{Introduction}

In last years seismic disturbances in VLF $(3-30 \mathrm{kHz}) / \mathrm{LF}$ (low band, $30-80 \mathrm{kHz}$ ) radio signals, as well as in LF (high band, $150-300 \mathrm{kHz}$ ) radio broadcasts, have been presented (Biagi et al., 2001a, b; Biagi and Hayakawa, 2002; Biagi et al., 2004, 2005, 2006, 2007; Gufeld et al., 1992; Hayakawa and Sato, 1994; Hayakawa et al., 1996, 2006; Molchanov and Hayakawa, 1998; Rozhnoi et al., 2005; Rozhnoi et al., 2006a, b). Recently, some possible seismic disturbances revealed by VLF radio signals collected on board of the French DEMETER satellite, were presented by Molchanov et al. (2006) and Rozhnoi et al. (2007). In any case, the disturbances in the VLF/LF radio signals seem to be produced by variations in the lower atmosphere/ionosphere of the area were the propagation of the radio signals happens. According to the most recent model (Mareev et al., 2002; Molchanov et al., 2006) these variations are due to turbulent atmospheric gravity waves of low amplitude that are generated near the ground surface by pre-seismic and postseismic processes such as gas and water releases in seismic active regions. The typical temporal scales of such processes range from some minutes to few hours and a time from 1 to $10 \mathrm{~h}$ needs for the energy to reach the lower atmosphere/ionosphere.

On 22 August 2005 an earthquake with $M=4.7$ occurred in the Anzio (central Italy) offshore area (Fig. 1). The epicenter is located 5 miles from the Tor Caldara "Diffuse Degassing Structure" where the ${ }^{222} \mathrm{Rn}$ and ${ }^{220} \mathrm{Rn}$ content in air is sampled by researchers of the INGV (National Institute of Geophysics and Volcanology). Before and after this earthquake,

Published by Copernicus Publications on behalf of the European Geosciences Union. 


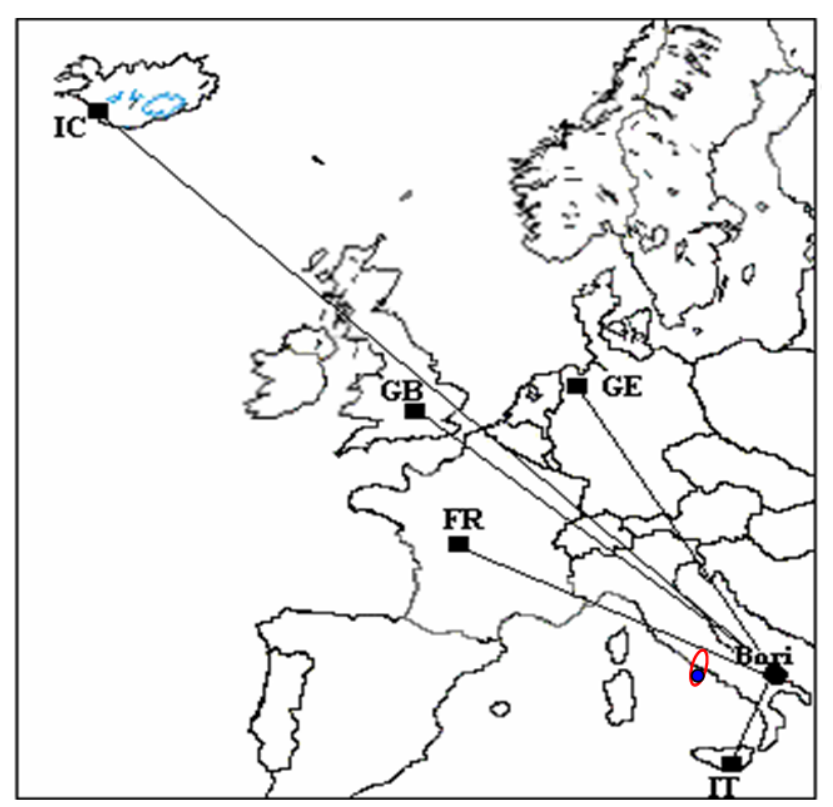

Fig. 1. The blue circle indicates the location of the 22 August 2005 Anzio earthquake $(M=4.7)$. The red ellipse roughly bounds the seismogenetic area in which the earthquake happened. The VLF/LF transmitters: GB ( $f=16 \mathrm{kHz}$, United Kingdom), FR ( $f=20.9 \mathrm{kHz}$, France), GE ( $f=23.4 \mathrm{kHz}$, Germany), IC ( $f=37.5 \mathrm{kHz}$, Iceland $)$, IT ( $f=54 \mathrm{kHz}$, Sicily, Italy) and the receiver (Bari) are shown in the map. The different radio-paths are indicated, too.

anomalies in the Rn content were revealed (Quattrocchi et al., 2007). The paths of the VLF/LF radio signals collected by a receiver working in Bari (south Italy) pass through the area where the epicenter of the earthquake is located (Fig. 1). Here the results of the study of possible seismic effects on the VLF/LF radio signals is presented.

\section{Data collecting and seismicity}

The VLF/LF receiver is located in the Department of Physics of Bari University and it is operating from 2002. The equipment is the Japanese OmniPAL model (Dowden and Adams, 1989) able to measure the electric field strength (intensity) and the phase of five VLF/LF radio signals. On the basis of the best reception at the receiver and taking into account the most convenient radio paths in relation to the seismic activity, the following transmitters were selected: GB ( $f=16 \mathrm{kHz}$, United Kingdom), FR $(f=20.9 \mathrm{kHz}$, France), GE ( $f=23.4 \mathrm{kHz}$, Germany), IC ( $f=37.5 \mathrm{kHz}$, Iceland $)$ and IT $(f=54 \mathrm{kHz}$, Sicily, Italy). The transmitters-receiver distance ranges from $500 \mathrm{~km}$ to $3500 \mathrm{~km}$. Figure 1 shows the location of the VLF-LF transmitters and of the receiver. A $5 \mathrm{~s}$ sampling rate was selected.

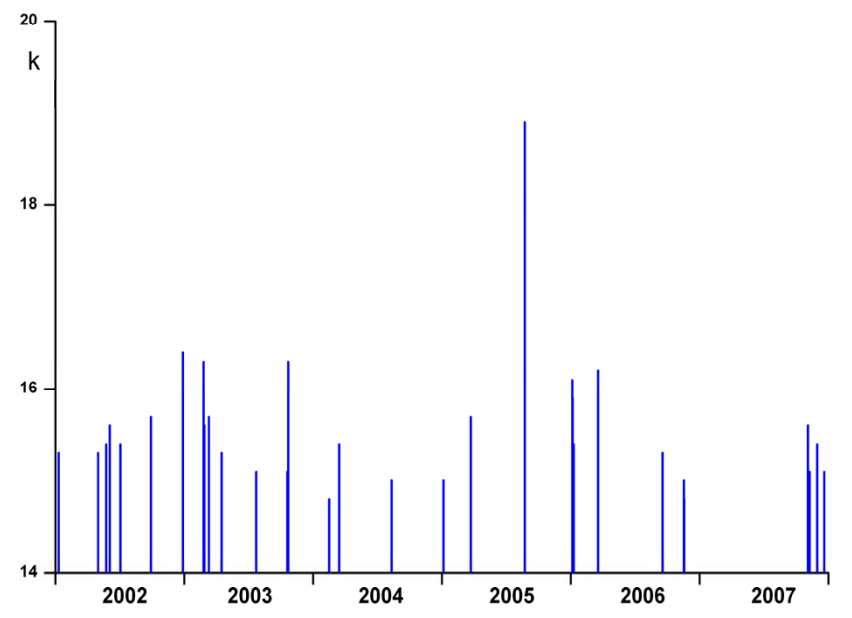

Fig. 2. Temporal trend of the $\mathrm{k}$ [energy $(\mathrm{erg})=10^{k}$ ] values of the earthquakes occurred in the seismogenetic area indicated in Fig. 1. The peak corresponds to the 22 August 2005 Anzio earthquake $(M=4.7)$.

The offshore Anzio earthquake (22 August 2005) was characterized by $M=4.7$ and a focal depth of $30 \mathrm{~km}$. The earthquake happened in a seismogenetic area, located near the coast of the Latium region (central Italy), that is approximately an ellipse, whose greater axis is $100 \mathrm{~km}$ long in the north-northeast direction (Fig. 1). The area is characterized by a moderate shallow seismic activity with $M$ in the range 2.0-3.5; so, the August 2005 earthquake was a rare event. To better validate this statement, the earthquakes occurred in this area from 2002 to 2007 were extracted from the Seismic Bulletins of the INGV. For each earthquake, the $k$ value [energy $\left.(\mathrm{erg})=10^{k}\right]$ was calculated from the magnitude $M$, using the relationship (Kanamori and Anderson, 1975):

$K=11.8+1.5 M$

The trend of the $\mathrm{k}$ values in the period 2002-2007 is reported in the Fig. 2 and the peak corresponding to the August 2005 earthquake stands up, clearly.

\section{Raw analysis}

Figure 3 shows the intensity raw data of the mentioned five radio signals collected during August 2005. The general features of the signals are the standard ones with high level at night time and low level at day time. The GB and IC signal are less disturbed than the other ones. From inspection of Fig. 3 it is evident that a short drop of the FR radio signal intensity happens on 19 August. In previous analyses (Kikuchi, 1981; Kleimenova et al., 2004; Rozhnoi et al., 2006b), a possible influence of the geomagnetic activity and of the meteorological conditions on the propagation of the VLF/LF radio signals have been revealed. So, at first, the geomagnetic activity and the meteorological conditions on the occasion of 


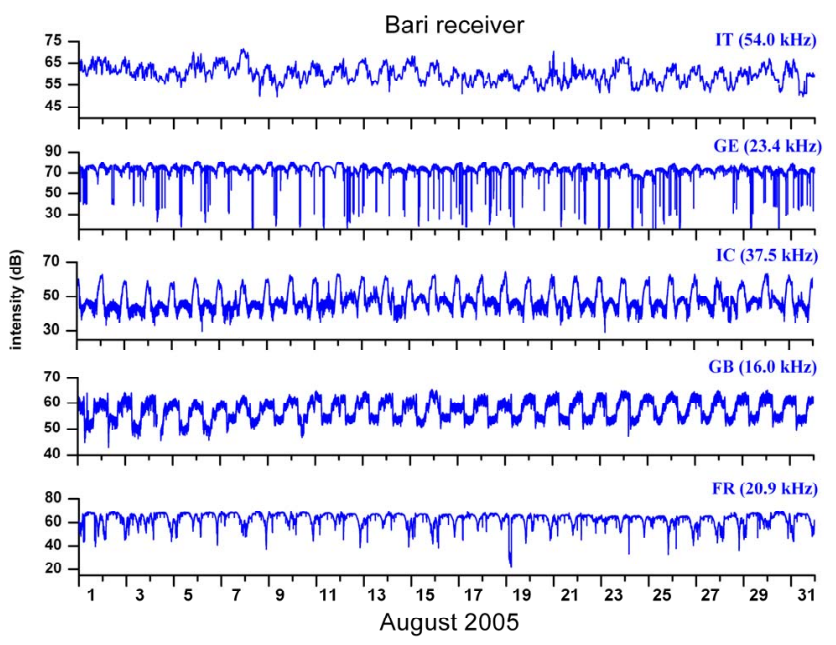

Fig. 3. The intensity (electric field strength) of the FR, GB, IC, GE and IT radio signals collected with a $5 \mathrm{~s}$ sampling rate by the Bari receiver during August 2005.

the previous radio intensity drop, were investigated. For the geomagnetic activity the $K_{p}$ and $D_{s t}$ indexes were considered. As for the meteorological conditions, the most sensitive zone is the one near the receiver where the signal is more weak and the effect of typhoons, cyclones or reflection surfaces and scattering zones produced in particular meteorological conditions, could produce more evident disturbances in the signals (Rozhnoi et al., 2006b). So, the data of a meteorological station located in Bari were used and the air temperature and the pressure were considered as the most indicative parameters. Figure 4 shows the geomagnetic-meteorological data and the FR intensity during August 2005. No correspondence among the 19 August radio intensity drop and meteorological and/or geomagnetic disturbances stands up. Then, in order to check the anomalous feature of the mentioned drop of the FR radio signal, the data collected by the Bari receiver have been strictly tested.

\section{Wavelet analysis}

As can be inferred by the inspection of the signal time series, its statistical characteristics change in time. A simple analysis based on the Fourier transform it is not the best tool for analyzing a non-stationary time series and hence a joint time-frequency analysis based on the wavelet transform (Torrence and Compo, 1998), has been applied. The wavelet method is capable of capturing simultaneously the time-varying nature of low frequency cycles and the frequency distribution of sudden and abrupt shocks in the original time series. In this way the use of variable-width time windows allows the localization of the signal in both time and frequency simultaneously. A picture, the spectrogram, showing both the amplitude of the signal versus the wavelet

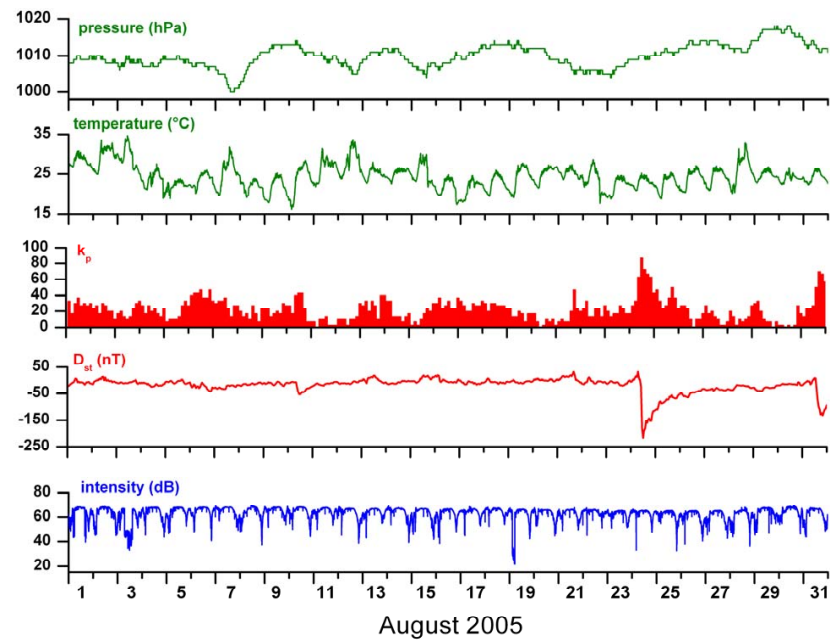

Fig. 4. During August 2005, from the top reading downwards: pressure, air temperature, $k_{p}$ geomagnetic index, $D_{s t}$ geomagnetic index and the intensity of the FR radio signal.

scale and the dependence between amplitude and time can be drawn (Daubechies, 1992; Strang and Nguyen, 1996).

In this study, the "Morlet function" has been considered as wavelet function (Torrence and Compo, 1998); this choice gives the best compromise as for a good resolution both in frequency and time. From a computational point of view, the radio signals raw data have not been considered, but the data obtained after a smoothing on 1 min basis. This procedure does not affect any spectral component whose period is greater than one minute. This smoothing was adopted in order to analyze at the same time more days and to avoid as much as possible the disturbances related to boundary effects. The main result of the wavelet analysis applied on the data of the radio signals intensity, is reported in Fig. 5 that is related to the interval 17-20 August 2005. Figure 5a clearly shows an increase in the power spectra in the band 60-120 min during 19 August on the FR radio signal; in Fig. $5 \mathrm{~b}$ the intensity of the spectrum, in arbitrary units, is reported by considering a cut along the horizontal dashed line in Fig. 5a. Here and in subsequent figures, the cut is chosen in correspondence with the maximum intensity in the two dimensional spectrum. The mentioned increase is an anomaly both with respect to the FR signal itself and with respect to the other radio signals. For example, in Fig. 6, the result of the analysis done on FR signal in the interval 13-16 August 2005 is reported. Taking into account that the colour scale automatically fits itself between the minimum and the maximum value of the spectra, Fig. 6 clearly shows that the intensity of the spectra is much lower with respect to the values of Fig. 5. As for an example of the other radio signals, the GE signal has been considered. The analysis done on this signal for different period and different years has shown that no anomaly could be claimed on 19 August 2005. A typical 

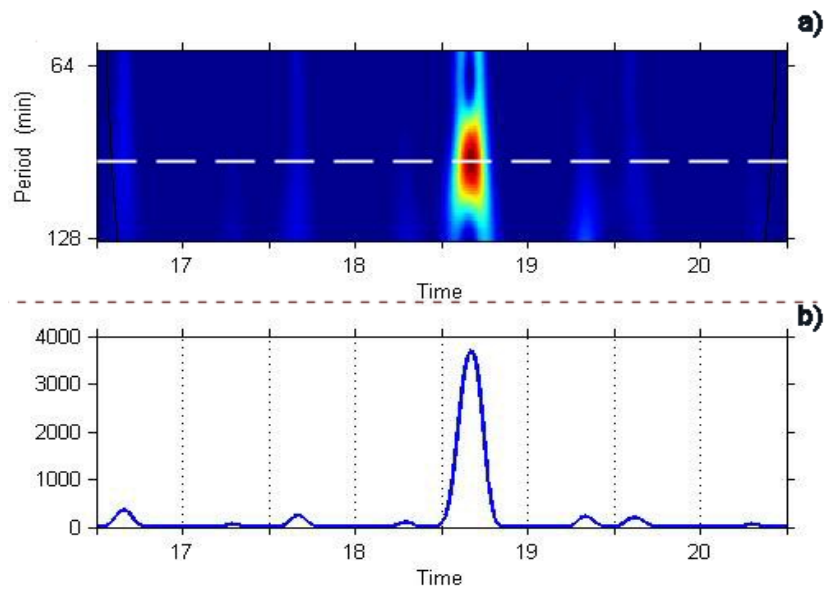

Fig. 5. (a) Normalized wavelet power spectrum from 17 to $20 \mathrm{Au}-$ gust 2005 for FR radio signal intensity in the range 64-128 min; the left axis is the Fourier period (in minute), the bottom axis is time and the wavelet power level (arbitrary scale) is represented with a colour scale from dark blue to dark red. (b) Plot of the intensity of the spectral component where maximum peak power appears corresponding to the white dashed line in (a) .
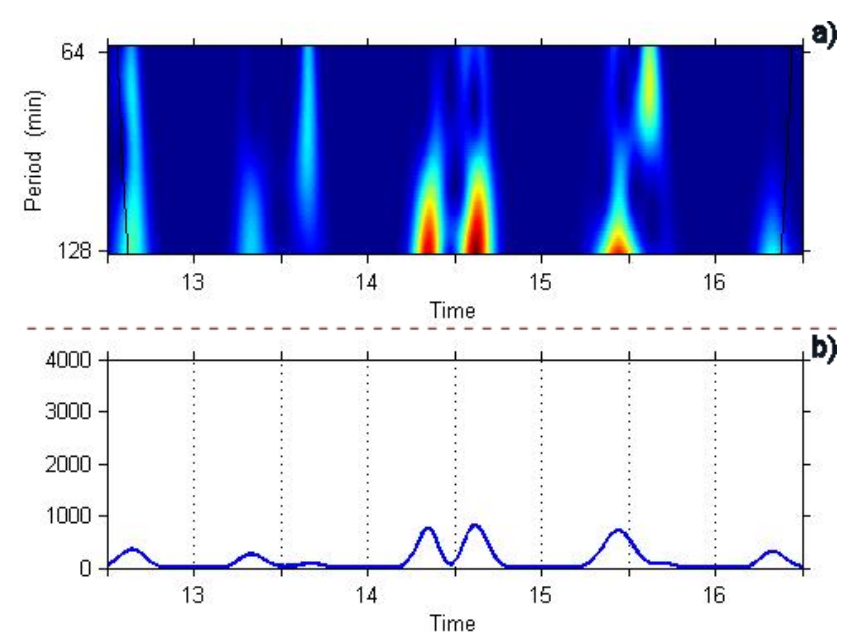

Fig. 6. (a) Normalized wavelet power spectrum from 13 to $16 \mathrm{Au}-$ gust 2005 for FR radio signal intensity in the range 64-128 min; the left axis is the Fourier period (in minute), the bottom axis is time and the wavelet power level (arbitrary scale) is represented with a colour scale from dark blue to dark red. (b) Plot of the intensity of the spectral component where maximum peak power appears.

example of analysis on the GE signal is shown in Fig. 7; daily, in the signal, a power spectrum increase in the band 60-120 min is present. Of course such regular increases cannot be considered anomalies (note that the intensity scale of GE signal is not comparable with that of FR signal).

Also the phase data have been analyzed with the same methodology. First of all, it must be noted that the phase signal itself is of a poor quality; it is much more affected by noise and shows much less regularity with respect to the in-

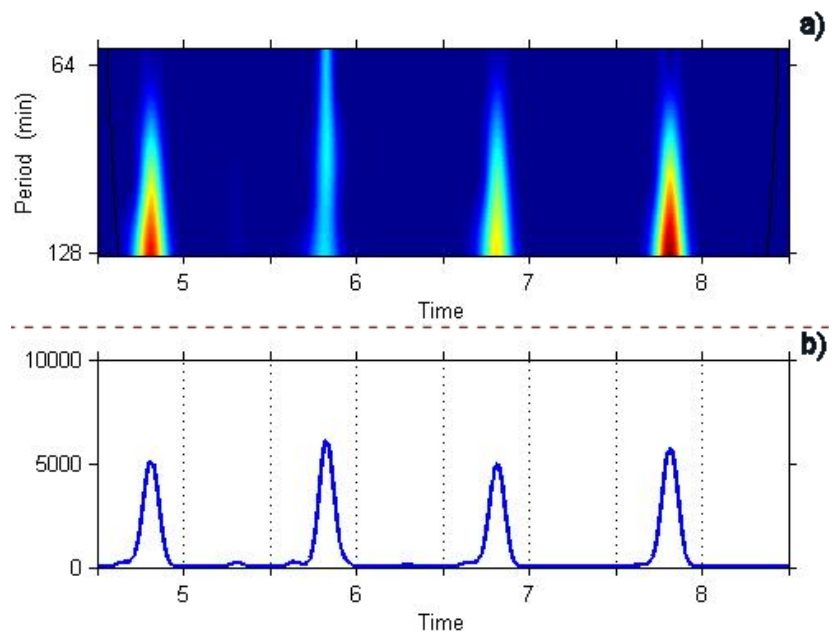

Fig. 7. (a) Normalized wavelet power spectrum from 5 to $8 \mathrm{Au}$ gust 2005 for GE radio signal intensity in the range 64-128 min; the left axis is the Fourier period (in minute), the bottom axis is time and the wavelet power level (arbitrary scale) is represented with a colour scale from dark blue to dark red. (b) Plot of the intensity of the spectral component where maximum peak power appears.

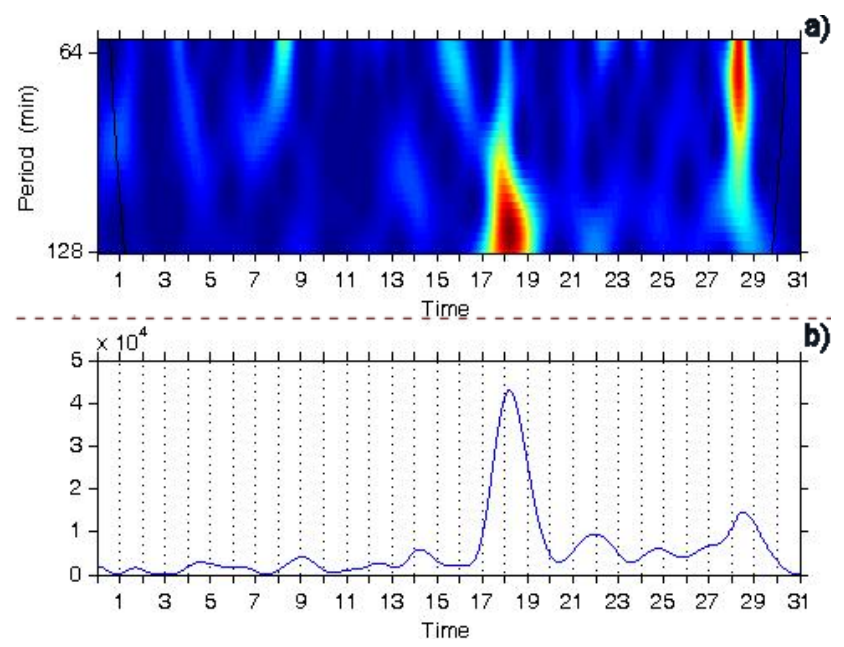

Fig. 8. (a) Normalized wavelet power spectrum for August 2005 for FR radio signal phase in the range 64-128 min; the left axis is the Fourier period (in minute), the bottom axis is time and the wavelet power level (arbitrary scale) is represented with a colour scale from dark blue to dark red. (b) Plot of the intensity of the spectral component where maximum peak power appears.

tensity signals. Nonetheless also the wavelet analysis of the FR phase signal shows an anomaly during 19 August 2005. Due to the different characteristics of the signal, the $10 \mathrm{~min}$ smoothed data have been considered; this allows to compute at the same time an entire month of data. In Fig. 8 the results of the wavelet analysis applied on the FR phase data during August 2005 are shown; the presence of the mentioned anomaly is evident 
a)

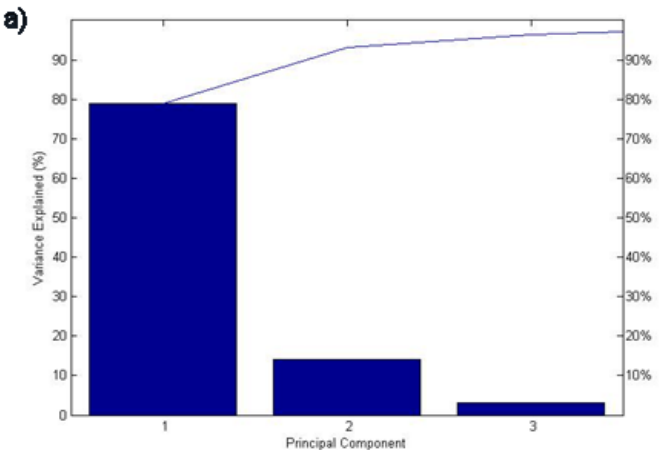

c)
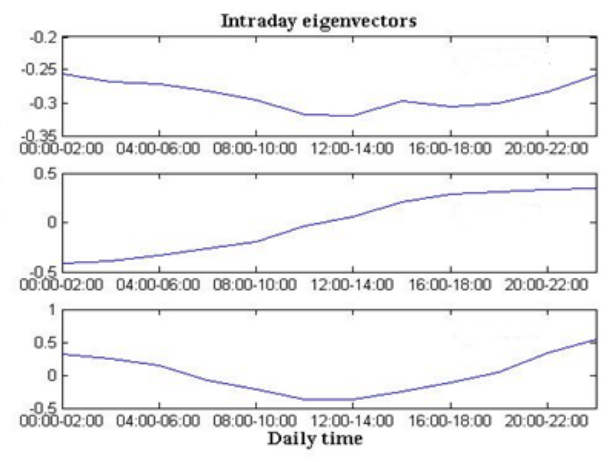

b)

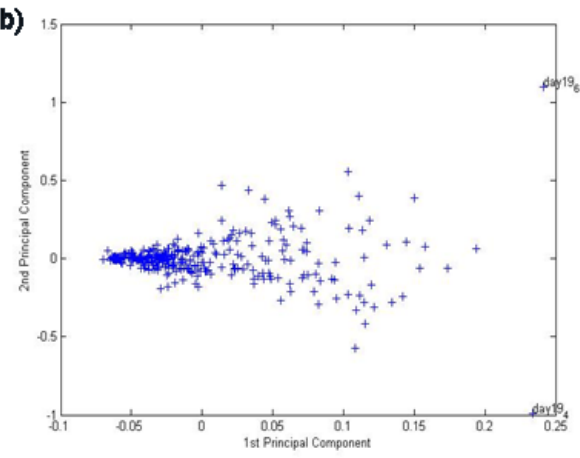

d)
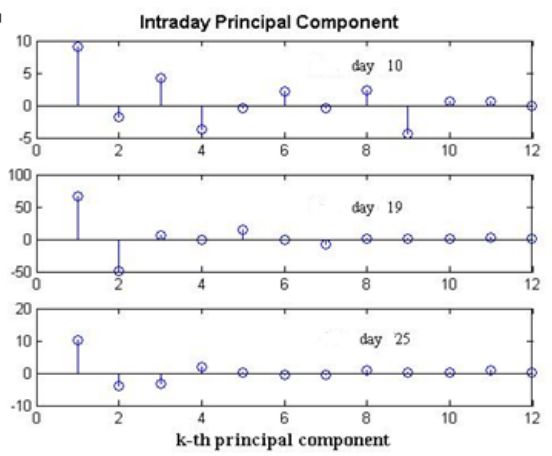

Fig. 9. (a) Boxplot of variance percentage explained by the first three principal components for the FR data. (b) August 2005 original FR data mapped into the new coordinate system defined by the first and second intraday principal components. Two outliers are evident and they are related to the 19 August, at 4 a.m. and at 6 a.m., respectively. (c) Intraday eigenvectors associated to the first eigenvalues explaining the maximum variance percentage. (d) Intraday principal components from days 10, 19 and 25 August 2005.

\section{Principal component analysis}

As further investigation, the principal component analysis (PCA) has been performed. This is a statistical technique whose purpose is to condense the information of a large set of correlated variables into a few variables (principal components), while not throwing overboard the variability present in the dataset (Joliffe, 2002). The principal components are derived as a linear combination of the variables of the dataset, with weights chosen so that the principal components become mutually uncorrelated. Each component contains new information about the dataset and it is ordered so that the first few components account for most of the variability. In a dataset processing, PCA takes its starting point from analyzing the length of the samples located in some suitable part of the set. By varying the length of the samples, the resolution of the analysis varies.

The radio signals sample is represented by the column vector $\boldsymbol{x}_{j}=\left[\boldsymbol{x}_{j}(1), \boldsymbol{x}_{j}(2), \ldots \ldots, \boldsymbol{x}_{j}(N)\right]^{T}$, where $N$ is the number of intervals investigated in one day; in such way, if $M$ is the ensemble of observations collected in one month, the entire radio signal monthly collected is represented by the $N \times M$ data matrix $\mathbf{X}=\left[\boldsymbol{x}_{1}, \boldsymbol{x}_{2}, \ldots ., \boldsymbol{x}_{M}\right]$. The derivation of the principal components is based on the assumption that the signal $\mathbf{X}$ is a zero-mean random process being characterized by the sample correlation matrix $\mathbf{R}_{\mathbf{X}}=\left(\mathbf{X X}^{T}\right) / M$. To obtain the whole set of $N$ different principal components, the following eigenvector/eigenvalue problem needs to be solved: $\mathbf{R}_{\mathbf{X}} \boldsymbol{w}=\lambda \boldsymbol{w}$, subject to the constraint $\|w\|=1$, where $w$ is the eigenvector and $\lambda$ is the eigenvalue associated. It is well known that the correlation matrix is real, symmetric and positive semi definite, so it can be factored into:

$\mathbf{R}_{\mathbf{X}}=W \Lambda W^{T}$

with the orthonormal eigenvectors in $\mathbf{W}$ and the eigenvalues in $\Lambda$ that are all grater than or equal to zero. The factorization in the relation (2) is provided by a singular value decomposition.

Here, on the basis of how long the raw anomaly under investigation lasted, the PCA analysis was applied setting the duration of each interval equal to $2 \mathrm{~h}$, that means $N=12$. So the signal sample $\boldsymbol{x}_{j}$ is a column vector of 12 components and $\mathbf{X}$ is a $12 \times 336$ matrix. It is worth noting that a month of 28 days (by leaving the first and last/second last day in the month) was considered in order to avoid edge effect that could bias the analysis.

The data of the FR signal were examined choosing the first three principal components; as shown in Fig. 9a these components explain $96.34 \%$ of variance. It is possible to observe the degree of morphologic day-by-day variability, looking at 

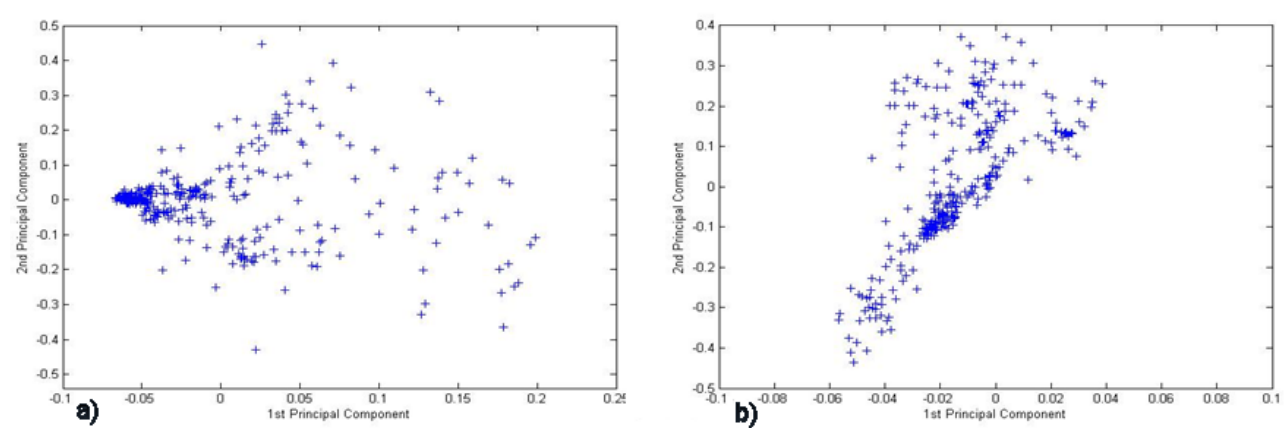

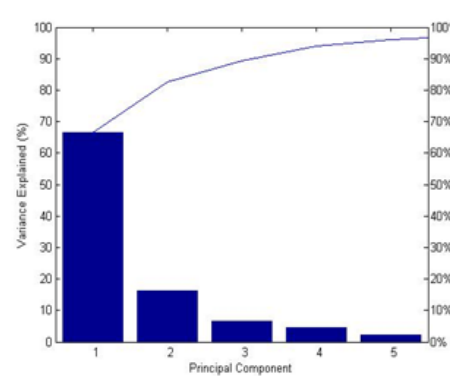

c)

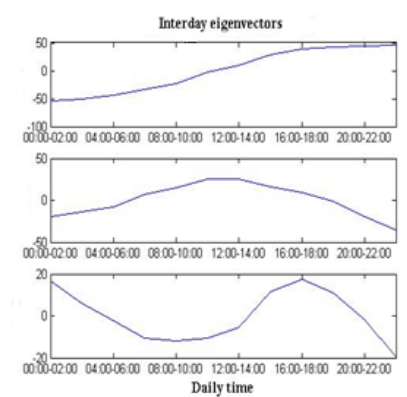

d)

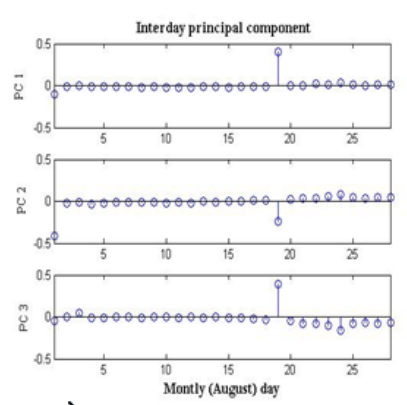

ө)

Fig. 10. (a) June 2004 original FR data mapped into the new coordinate system defined by the first and second intraday principal components. No outliers stand up and the data appear like a compact cloud of points. (b) June 2004 original GE data mapped into the new coordinate system defined by the first and second intraday principal components. No outliers appear and the data appear like a compact cloud of points. (c) Boxplot of variance percentage explained by the first five interday principal components for the FR data. (d) The first three interday eigenvectors observed during $24 \mathrm{~h}$. (e) The first three interday principal components observed during August 2005.

the plot in Fig. 9b that represents the first principal component (PC1) versus the second one (PC2). Here two outliers appear and they define an anomaly occurring on $19 \mathrm{Au}-$ gust 2005 between 4 and 6 a.m. This behaviour is anomalous both respect to the FR data and to the data of the other radio signals collected. To this end, as an example, Fig. 10a related to FR June 2004 data and Fig. 10b related to GE June 2005 data, both projected on PC1 and PC2 can be observed. In these Figures no outliers appear like in Fig. 9b; the original data, mapped into the new coordinate system defined by the first two principal components, are displayed like a compact cloud of points in the direction of maximum variance.

The trend of eigenvectors associated to the three most informative eigenvalues is reported in Fig. 9c. The first and third eigenvectors seem to follow the night-day time variations; the second eigenvector, instead, seems to take into account the trend of the signal anomaly. Finally, it's worth noting that by analyzing all the principal components from each day (Fig. 9d) it is possible to see that a strong variation comes out the day 19 August, confirming the presence of a signal anomaly with respect to the other days. The presented results characterize the "intraday" correlation obtained by the sample correlation matrix $\mathbf{R}_{\mathbf{X}}$, as defined before. Also another correlation matrix $\mathbf{R}^{\prime}{ }_{\mathbf{X}}=\left(\mathbf{X}^{T} \mathbf{X}\right) / N$ characterizing the "interday" correlation, was defined and used. On the basis of $89.31 \%$ variance explained (Fig, 10c), three principal components were selected. These components reported in Fig. 10e, show a clear anomaly in the FR data occurring on 19 August. In this case, PC1 seems to take into account the trend of the signal anomaly and PC2-PC3 seem to follow the night-day time variations, as Fig. 10d shows.

\section{Discussion}

The wavelet and PCA analyses presented in the previous sessions have clearly confirmed the result of the raw analysis, i.e. on 19 August 2005 one anomaly exists in the FR radio signal collected by the Bari receiver. Both these analyses have proved useful to point out the anomaly and no particular advantage of one tool respect the other one was revealed.

The characteristics of the mentioned anomaly are: a) its appearance only on the FR signal, b) its existence both in the intensity and the phase data, c) a duration of few hours.

No connection of the anomaly with the geomagnetic activity or the meteorological condition stands up. On the contrary, the radio anomaly appears three days prior the occurrence of the 22 August earthquake $(M=4.7)$ and from Fig. 1 it is evident that the FR radio path is the nearest to the epicentre. More precisely, it was verified that the epicenter is inside the 5th Fresnel zone of the FR-Bari path. It must be noted that the quoted zone is considered to be the most sensitive 
to the seismo-atmospheric effects (Hayakawa et al., 1996; Molchanov and Hayakawa, 1998; Rozhnoi et al., 2005).

This earthquake was a rare event because its magnitude was large with respect to the normal seismic activity of the relative seismogenetic zone. Besides, the anomalies in the air $\mathrm{Rn}$ content revealed before the occurrence of the earthquake (paragraph 1) indicate that pre-seismic processes took place.

On the basis of these statements, the radio anomaly under study appears clearly a precursor of the earthquake. The duration of the anomaly fits with the characteristic periods of the Atmospheric Gravity Waves, so that disturbances in the ionosphere turbulence produced by an upward energy flux of atmospheric gravity waves which is induced by processes occurring during the preparation of the earthquake, can give justification to its existence.

\section{Conclusions}

A preseismic anomaly, with a duration of few hours, was revealed three days prior the occurrence of the offshore Anzio earthquake ( $M=4.7 ; 22$ August 2005) in a VLF radio signal. Among the five radio signals recorded by the receiver, the anomaly appeared only in the signal (FR), the 5th Fresnel zone of which contains the epicenter. This result is another evidence of the information on the location of a future earthquake that the anomalies in the propagation of the VLF/LF radio signals can provide.

Acknowledgements. This research was conducted in the framework of the Cooperation Agreement between the University of Bari (Italy) and the Institute of the Earth's Physics, Russian Academy of Sciences.

The seismic data used in this paper were extracted by the INGV (Istituto Nazionale di Geofisica e Vulcanologia) web site (http://www.ingv.it). The geomagnetic data were obtained by web site: www.ngdc.noaa.gov, Geomagnetism Home, National Geophysical Data Center.

Edited by: M. Contadakis

Reviewed by: M. Y. Boudjada and V. Lapenna

\section{References}

Biagi, P. F., Piccolo, R., Ermini, A., Martellucci, S., Bellecci, C., Hayakawa, M., Capozzi, V., and Kingsley, S. P.: Possible earthquake precursors revealed by LF radio signals, Nat. Hazards Earth Syst. Sci., 1, 99-104, 2001a, http://www.nat-hazards-earth-syst-sci.net/1/99/2001/.

Biagi, P. F., Piccolo, R., Ermini, A., Martellucci, S., Bellocci, C., Hayakawa, M., and Kingsley, S. P.: Disturbances in LF radio-signals as seismic precursors, Annali di Geofisica, 44, 5/6, $2001 b$.

Biagi, P. F. and Hayakawa, M.: Possible premonitory behaviour of LF radiowaves on the occasion of the Slovenia earthquakes $(M=5.2-6.0-5.1)$ occurred on March-May 1998, in: Seismo Electromagnetics: Lithosphere-Atmosphere-Ionosphere
Coupling, edited by: Hayakawa, M. and Molchanov, O., TERRAPUB, Tokyo, 249-253, 2002.

Biagi, P. F., Piccolo, R., Castellana, L., Maggipinto, T., Ermini, A., Martellucci, S., Bellecci, C., Perna, G., Capozzi, V., Molchanov, O. A., Hayakawa, M., and Ohta, K.: VLF-LF radio signals collected at Bari (South Italy): a preliminary analysis on signal anomalies associated with earthquakes, Nat. Hazards Earth Syst. Sci., 4, 685-689, 2004,

http://www.nat-hazards-earth-syst-sci.net/4/685/2004/.

Biagi, P. F., Castellana, L., Maggipinto, T., Piccolo, R., Minafra, A., Ermini, A., Martellucci, S., Bellecci, C., Perna, G., Capozzi, V., Molchanov, O. A., and Hayakawa, M.: A possible preseismic anomaly in the ground wave of a radio broadcasting $(216 \mathrm{kHz})$ during July-August 1998 (Italy), Nat. Hazards Earth Syst. Sci., 5, 727-732, 2005, http://www.nat-hazards-earth-syst-sci.net/5/727/2005/.

Biagi, P. F., Castellana, L., Maggipinto, T., Piccolo, R., Minafra, A., Ermini, A., Martellucci, S., Bellecci, C., Perna, G., Capozzi, V., Molchanov, O. A., and Hayakawa, M.: LF radio anomalies revealed in Italy by the wavelet analysis: Possibile preseismic effects during 1997-1998, Phys. Chem. Earth, 31, 403-408, 2006.

Biagi, P. F., Castellana, L., Maggipinto, T., Maggipinto, G., Minafra, A., Ermini, A., Capozzi, V., Perna, G., Solovieva, M., Rozhnoi, A., Molchanov, O. A., and Hayakawa, M.: Decrease in the electric intensity of VLF/LF radio signals and possible connections, Nat. Hazards Earth Syst. Sci., 7, 423-430, 2007, http://www.nat-hazards-earth-syst-sci.net/7/423/2007/.

Daubechies, I.: Ten lectures on wavelets, in: CBMS - NSF Regional Conferences Series in Applied Mathematics SIAM, Philadelphia PA, 61, 1992

Dowden, R. L. and Adams, C. D.: Phase and amplitude perturbations on the NWC signal at Dunedin form lightning-induced electron percipitation, J. Geophys. Res., 94, 497-503, 1989.

Gufeld, I. L., Rozhnoi, A. A., Tyumensev, S. N., Sherstuk, S. V., and Yampolsky, V. S.: Radiowave disturbances in period to Rudber and Rachinsk earthquakes, Phys. Solid Earth, 28(3), 267-270, 1992.

Hayakawa, M. and Sato, H.: Ionospheric perturbations associated with earthquakes, as detected by subionospheric VLF propagation, in: Electromagnetic Phenomena Related to Earthquake Prediction, edited by: Hayakawa, M. and Fujinawa, Y., TERRAPUB, Tokyo, 391-397, 1994.

Hayakawa, M., Molchanov, O. A., Ondoh, T., and Kawai, E.: The precursory signature effect of the Kobe earthquake on subionospheric VLF signals, J. Comm. Res. Lab., 43, 169-180, 1996.

Hayakawa, M., Ohta, K., Maekawa, S., Yamauchi, T., Ida, Y., Gotoh, T., Yonaiguchi, N., Sasaki, H., and Nakamura, T.: Electromagnetic precursors to the 2004 Mid Niigata Prefecture earthquake, Phys. Chem. Earth, 31, 356-364, 2006.

Kanamori, H. and Anderson, D.: Theoretical basis of some empirical relations in seismology, Bull. Seismol. Soc. Am., 65, 1073 1095, 1975.

Kikuchi, T.: VLF phase anomalies associated with substorm, Mem. Nat. Inst. Polar. Res. (Special Issue), 18, 3-23, 1981.

Kleimenova, N. G., Kozyreva, O. V., Rozhnoi, A. A., and Solovieva, M. S.: Variation in the VLF signal parameters on the AustraliaKamchatka radio path during magnetic storms, Geomagnet. Aeronomy, 44(3), 354-361, 2004.

Joliffe, I. T.: Principal Component Analysis, Springer, New York, 
NY, USA, 2002.

Mareev, E. A., Iudin, D. I., and Molchanov, O. A.: Mosaic source of internal gravity waves associated with seismic activity, in: Seismo Electromagnetics: Lithosphere-Atmosphere-Ionosphere Coupling, edited by: Hayakawa, M. and Molchanov, O., TERRAPUB, Tokyo, 249-253, 2002.

Molchanov, O. A. and Hayakawa, M.: Subionospheric VLF signal perturbations possibly related to earthquakes, J. Geophys. Res., 103, 17 489-17 504, 1998.

Molchanov, O., Rozhnoi, A., Solovieva, M., Akentieva, O., Berthelier, J. J., Parrot, M., Lefeuvre, F., Biagi, P. F., Castellana, L., and Hayakawa, M.: Global diagnostics of the ionospheric perturbations related to the seismic activity using the VLF radio signals collected on the DEMETER satellite, Nat. Hazards Earth Syst. Sci., 6, 745-753, 2006,

http://www.nat-hazards-earth-syst-sci.net/6/745/2006/.

Quattrocchi, F., Voltattorni, N., Cantucci, B., Cinti, D., Gasparini, C., Pizzino, L., and Procesi M.: The Tor Caldara $\mathrm{CO}_{2}$ "Diffuse Degassing Structure (DDS)" in the Alban Hills seismotectonic framework: ${ }^{222} \mathrm{Rn} /{ }^{220} \mathrm{Rn}$ output before and after the $22 \mathrm{Au}$ gust 2005 Anzio Earthquake $\left(M_{w}=4.6\right)$, IUGG2007 Abstracts Book, 2007.
Rozhnoi, A. A., Solovieva, M. S., Molchanov, O. A., Hayakawa, M., Maekawa, S., and Biagi, P. F.: Anomalies of LF signal during seismic activity in November-December 2004, Nat. Hazards Earth Syst. Sci., 5, 657-660, 2005, http://www.nat-hazards-earth-syst-sci.net/5/657/2005/.

Rozhnoi, A. A., Solovieva, M. S., Molchanov, O. A., Chebrov, V., Voropaev, V., Hayakawa, M., Maekawa, S., and Biagi, P. F.: Preseismic anomaly of LF signal on the wave path Japan-Kamchatka during November 2004, Phys. Chem. Earth, 31, 422-427, 2006 a.

Rozhnoi, A., Solovieva, M. S., Molchanov, O. A., Hayakawa, M., Maekawa, S. and Biagi, P.F.: Sensitivity of LF signal to global ionosphere and atmosphere perturbations in the network of stations, Phys. Chem. Earth, 31, 409-415, 2006 b.

Rozhnoi, A., Molchanov, O., Solovieva, M., Gladyshev, V., Akentieva, O., Berthelier, J. J., Parrot, M., Lefeuvre, F., Hayakawa, M., Castellana, L., and Biagi, P. F.: Possible seismo-ionosphere perturbations revealed by VLF signals collected on ground and on a satellite, Nat. Hazards Earth Syst. Sci., 7, 617-624, 2007, http://www.nat-hazards-earth-syst-sci.net/7/617/2007/.

Strang, G. and Nguyen, T.: Wavelets and filter banks, Wellesley Cambridge Press, 490 pp., 1996.

Torrence, C. and Compo, G. P.: A practical Guide to Wavelet Analysis, Bull. American Meteorol. Soc., 79(1), 61-78, 1998. 